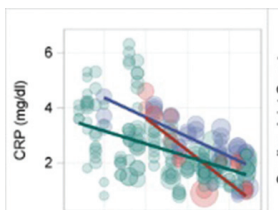

2000200520102015

Year

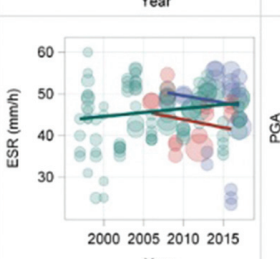

Year

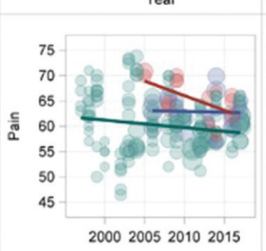

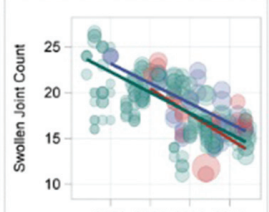
Year

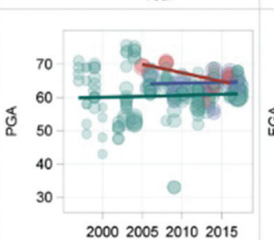

Year

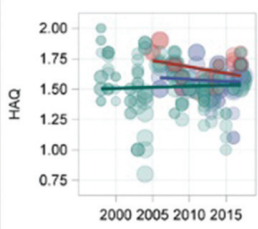

Yea 2000200520102015

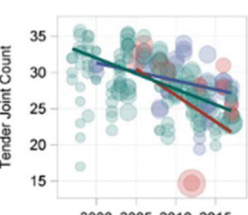

Year

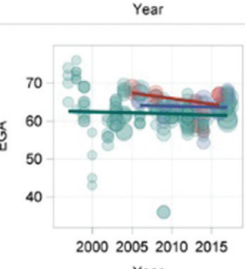

Year

\section{DMARD naive} bDMARD IR CSDMARD IR

Size of bubbles represent

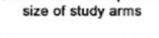

Lines represent weighted
linear regression lines

Conclusions: There is a progressive drift towards lower number of swollen and tender joints and lower CRP-levels at trial entry of time, which is at least partly related to a similar trend in inclusion criteria for RA. The constancy of patientreported outcomes suggests that the baseline activity is still perceived as similarly high. Differences in overall baseline inflammatory activity may pose a challenge for comparing newer with older trial results.

Disclosure of Interest: None declared

DOI: 10.1136/annrheumdis-2018-eular.6657

\section{SAT0689 THYMECTOMY IN PATIENTS WITH MYASTHENIA GRAVIS AND THE RISK OF AUTOIMMUNE RHEUMATIC DISEASES: A NATIONWIDE COHORT STUDY}

C.C. Chang ${ }^{1,2}$, J.-H. Chen ${ }^{3}$. ${ }^{1}$ Division of Allergy, Immunology and Rheumatology, Department of Internal Medicine, School of Medicine, College of Medicine, Taipei Mediacal University, ${ }^{2}$ Division of Rheumatology, Immunology and Allergy, Department of Internal Medicine, Taipei Medcial Universuty Hospital; ${ }^{3}$ Biostatistics Center and Graduate Institute of Data Science, College of Management, Taipei Medical University, Taipei, Taiwan, Province of China

Background: Previous studies have shown myasthenia gravis (MG) and autoimmune rheumatic diseases (ARDs) share common pathogenetic mechanisms. Objectives: Therefore, the present study investigated the possible relationship between MG and ARDs.

Methods: We analysed Taiwanese medical data from the Registry of Catastrophic Illness and identified patients with MG. From the entire general population data of the National Health Insurance Research Database, we randomly selected a comparison cohort that was frequency-matched by age (in 5 year increments), sex, and index date. We analysed the risk of ARDs by using a Cox proportional hazards regression model stratified by sex, age, and treatment.

Results: We enrolled 6478 patients with MG (58.03\% women; mean age, 50.55 years) and 25912 age- and sex-matched controls in the present study. The risk of total ARDs was 6.25 times higher in the MG cohort than in the non-MG cohort after adjustment for age and sex. Furthermore, the MG cohort was associated with a significantly higher risk of primary SS (pSS), SLE, and other ARD types (adjusted hazard ratios [HRs]: 15.84 [95\% Cl: 8.39-23.91]; 11.32 [95\% Cl: 5.04-25.429]; and 4.07 [95\% Cl: 1.31-12.62], respectively). MG cohort who received thymectomy had an increased risk of RA, pSS, and SLE (adjusted HRs: 4.41; 15.06; and 23.68, respectively).
Conclusions: The present nationwide cohort study revealed an association between MG and incident ARDs. MG cohort who received thymectomy had an increased risk of RA, pSS, and SLE. Future studies are needed to elucidate the underlying pathogenesis and to translate them into clinical therapeutic options.

\section{REFERENCES :}

[1] Voight BF, Cotsapas C. Human genetics offers an emerging picture of common pathways and mechanisms in autoimmunity.Curr Opin Immunol 2012;24:552-557.

[2] Parkes M, Cortes A, van Heel DA, Brown MA. Genetic insights into common pathways and complex relationships among immune-mediated diseases. Nat Rev Genet 2013;14:661-673

[3] Fang F, Sveinsson O, Thormar G, et al. The autoimmune spectrum of myasthenia gravis: a Swedish population-based study. J Intern Med 2015;277(5):594-604.

[4] Christensen PB, Jensen TS, Tsiropoulos I, et al. Autoimmune diseases in myasthenia gravis. A population-based study Acta Neurol Scand 1995;91 (3):192-195.

Disclosure of Interest: None declared

DOI: 10.1136/annrheumdis-2018-eular.4375

\section{SAT0690 THE ASSOCIATION BETWEEN SARCOIDOSIS AND ISCHAEMIC HEART DISEASE - A BIG DATA ANALYSIS}

D. Katz ${ }^{1,2}$, S. Tiosano ${ }^{3,4}$, D. Comaneshter ${ }^{5}$, A.D. Cohen ${ }^{5,6}$, H. Amital ${ }^{7,8}$.

${ }^{1}$ Zabludowicz Center for Autoimmune Diseases, Sheba Medical Center, Tel Hashomer, Ramat Gan; ${ }^{2}$ Faculty of Medicine, Hebrew University of Jerusalem, Jerusalem; ${ }^{3}$ Department of Medicine ' $B$ ' and Zabludowicz Center for Autoimmune Diseases, Sheba Medical Center, Tel-Hashomer, Ramat Gan; ${ }^{4}$ Sackler Faculty of Medicine, Tel-Aviv University; ${ }^{5}$ Chief Physician's Office, Clalit Health Services, Tel Aviv, ${ }^{6}$ Siaal Research Center for Family Medicine and Primary Care, Faculty of Health Sciences, Ben Gurion University of the Negev, Beer Sheva; ${ }^{7}$ Department of Medicine ' $B$ ' and Zabludowicz Center for Autoimmune Diseases, Sheba Medical Center, Tel-Hashomer, Ramat Gan; ${ }^{8}$ Sackler Faculty of Medicine, Tel-Aviv University, Tel Aviv, Israel

Background: Sarcoidosis is an inflammatory disease characterised by the hallmark sign of non-caseating granulomas ${ }^{1,2}$. In the past decade a consensus has formed regarding the pivotal role of inflammation in atherosclerosis ${ }^{3}$. Since this discovery the association between chronic inflammatory states and ischaemic heart disease was confirmed in several rheumatic diseases ${ }^{4}$. Therefore, the constant state of inflammation to which sarcoidosis patients are exposed might pose as a risk factor for ischaemic heart disease.

Objectives: The aim of this study is to assess the relation between sarcoidosis and Ischaemic heart disease and its prognostic significance.

Methods: Based on data from Clalit Health Services (CHS), Israel's largest health maintenance organisation, the medical records of 3993 sarcoidosis patients and 19856 controls were acquired. Controls were matched to sarcoidosis patients according to age and sex. Chi-square and student t-tests were used in order to compare variables distribution in the cohort. Variables associated with ischaemic heart disease were assessed by logistic regression model. Log-rank test was performed for survival analysis, while Cox proportional hazards method was utilised to evaluate variables related to increased risk of all-cause mortality.

Results: Matched by sex and age - both sarcoidosis group and the control group were composed from $63 \%$ females with mean age being 56 years. Compared to the control group, sarcoidosis patients had a higher proportion of ischaemic heart disease, presenting with $856(21.4 \%)$ cases whereas the control group had only 2999 cases $(15.1 \%, p<0.001)$. The association between sarcoidosis and ischae mic heart disease was demonstrated by a multivariate analysis, (adjusted OR $1.503,95 \% \mathrm{Cl} 1.361-1.660)$. A 15 year follow up revealed increased mortality among sarcoidosis patients - as $710(17.8 \%)$ of sarcoidosis patients had passed away while $2121(10.7 \%)$ deaths were reported in the control group $(p<0.001)$. In a multivariate model, sarcoidosis patients were found to be in increased risk for all-cause mortality compared to the control group (adjusted HR 1.95, 95\% Cl 1.75-2.14). 